\title{
Liquid Crystalline Phase Behavior of Protein Fibers in Water: Experiments versus Theory
}

\author{
Jin-Mi Jung ${ }^{\dagger}$ and Raffaele Mezzenga*,†,* \\ ${ }^{\dagger}$ Department of Physics and FRIMAT Center for Nanomaterials, University of Fribourg, Fribourg CH-1700, \\ Switzerland, and Department of Food Science and Technology, Nestlé Research Center, Vers-chez-les-Blanc, \\ Lausanne 26, CH-1000, Switzerland
}

\begin{abstract}
We have developed a new method allowing the study of the thermodynamic phase behavior of mesoscopic colloidal systems consisting of amyloid protein fibers in water, obtained by heat denaturation and aggregation of $\beta$-lactoglobulin, a dairy protein. The fibers have a cross section of about $5.2 \mathrm{~nm}$ and two groups of polydisperse contour lengths: (i) long fibers of $1-20 \mu \mathrm{m}$, showing semiflexible behavior, and (ii) short rods of 100-200 nm long, obtained by cutting the long fibers via high-pressure homogenization. At $\mathrm{pH} 2$ without salt, these fibers are highly charged and stable in water. We have studied the isotropic-nematic phase transition for both systems and compared our results with the theoretical values predicted by Onsager's theory. The experimentally measured isotropic-nematic phase transition was found to occur at $0.4 \%$ and at $3 \%$ for the long and short fibers, respectively. For both systems, this phase transition occurs at concentrations more than 1 order of magnitude lower than what is expected based on Onsager's theory. Moreover, at low enough $\mathrm{pH}$, no intermediate biphasic region was observed between the isotropic phase and the nematic phase. The phase diagrams of both systems ( $\mathrm{pH}$ vs concentration) showed similar, yet complex and rich, phase behavior. We discuss the possible physical fundamentals ruling the phase diagram as well as the discrepancy we observe for the isotropic-nematic phase transition between our experimental results and the predicted theoretical results. Our work highlights that systems formed by water-amyloid protein fibers are way too complex to be understood based solely on Onsager's theories. Experimental results are revisited in terms of the Flory's theory (1956) for suspensions of rods, which allows accounting for rod-solvent hydrophobic interactions. This theoretical approach allows explaining, on a semiquantitative basis, most of the discrepancies observed between the experimental results and Onsager's predictions. The sources of protein fibers complex colloidal behavior are analyzed and discussed at length.
\end{abstract}

\section{Introduction}

Lyotropic liquid crystals are encountered in a multitude of biological systems and have both fundamental and practical relevance, since they can be used in a number of applications such as cosmetic, pharmaceutical, medical, or nutrition technologies. ${ }^{1,2}$

Possibly the type of liquid crystalline system most frequently found is that based on anisotropic particles dispersed in a liquid. Differently from spherical particles, the phase behavior of anisotropic particles can be rich due to the possibility of orientational ordering. This orientational ordering occurs at a critical volume fraction, when the particles go from an isotropic to a nematic distribution in the solvent. This transition originates from a competition between two types of entropy: the orientational entropy, which is dominant at low concentration and is maximized by an isotropic distribution, and the packing (or excluded volume) entropy, which becomes more important at high concentration and favors a nematic distribution. The resulting gain of the free volume with progressive increase of concentrations compensates for the loss of orientational entropy, minimizing the total free energy.

In 1949, Onsager constructed an elegant theory predicting the phase behavior of anisotropic particles based on excluded volume

*Corresponding author. E-mail: raffaele.mezzenga@unifr.ch, raffaele. mezzenga@rdls.nestle.com.

(1) Mezzenga, R.; Schurtenberger, P.; Burbidge, A.; Michel, M. Nat. Mater. $2005,4,729-740$.

(2) Sagalowicz, L.; Mezzenga, R.; Leser, M. E. Curr. Opin. Colloid Interface Sci. 2006, 11, 224-229. interactions and proposed it in a seminal paper. ${ }^{3}$ He showed that the isotropic-nematic transition for stiff slender particles could be treated within a virial expansion of the total free energy. Although his theory has an analytical solution only in the limit of aspect ratios $L / D \rightarrow \infty$, for very thin rigid hard particles with high enough aspect ratio, the virial expansion can be truncated after the second virial term, making it particularly suitable to determine isotropic-nematic transition in practical cases and real systems. This transition was shown to be a first-order thermodynamic transition and to be purely athermal, because originating by the minimization of purely entropic energetic terms. An intermediate isotropic-nematic biphasic region was also predicted to exist in between the pure isotropic and nematic phases. This theory has become very popular over the past six decades and has been extended with the years to shorter particles, polydisperse particles, charged particles, and semiflexible particles. ${ }^{3-5}$

Numerous model systems of anisotropic particles in a solvent were investigated and compared to Onsager's theory, showing often a good agreement with the predicted results. These anisotropic particles covered a wide range of fields, ranging from inorganic to organic and also biological particles, such as tobacco mosaic virus. ${ }^{6,7}$ An extensive list of the systems studied and

(3) Onsager, L. Ann. N.Y. Acad. Sci. 1949, 51, 627-659.

(4) Stroobants, A.; Lekkerkerker, H. N. W.; Odijk, Th. Macromolecules 1986, $19,2232-2238$.

(5) Vroege, G. J.; Lekkerkerker, H. N. W. Rep. Prog. Phys. 1992, 55, 1241-1309.

(6) Purdy, K. R.; Varga, S.; Galindo, A.; Jackson, G.; Fraden, S. Phys. Rev. Lett. 2005, 94, 057801-1-057801-4.

(7) Purdy, K. R.; Fraden, S. Phys. Rev. E: Stat., Nonliear, Soft Matter Phys. 2004, 70, 061703-1-061703-8. 
benchmarked to Onsager's theory can be found in brilliant reviews devoted to the field. ${ }^{4,8}$

Food protein fibers in water, obtained by heat denaturation and aggregation of $\beta$-lactoglobulin, a whey protein which is widely used in food industries, ${ }^{9,10}$ are an interesting system to test isotropic-nematic transitions in water, as the relevance of this system clearly goes beyond purely academic purposes. $\beta$-Lactoglobulin has 162 amino acids, a molecular weight of $18.3 \mathrm{kDa}$, and a compact globular structure of $4 \mathrm{~nm}$ of diameter, with a hydrophobic core. This protein is known to form heatinduced aggregates of various structures and morphologies depending on the concentration, the $\mathrm{pH}$, ionic strength of water, the temperature, and the heating time. ${ }^{11-15}$ In particular, long, thin, and rodlike aggregates can be obtained from heat denaturation and aggregation of $\beta$-lactoglobulin at low $\mathrm{pH}$ and low ionic strength. ${ }^{9,11,12,16-18}$ The ability to form these irreversible fibrils, ${ }^{19}$ formed through noncovalent bonding, ${ }^{20}$ can then be exploited in order to obtain a real system of food-grade anisotropic fibers in water.

Phase diagrams of $\beta$-lactoglobulin rodlike aggregates built from heated solutions of native protein with varying initial concentrations, $\mathrm{pH}$, and ionic strength have already been attempted in the literature. ${ }^{17,21,22}$ In all formerly investigated systems, however, a coexistence of fibers with supramolecular aggregates called spherulites was observed. ${ }^{10,17,21,23}$ These spherulites are formed simultaneously with the fibers, and their number and size depend on the initial concentration. Because spherulites do not dissolve upon dilution in water, they clearly constitute nonequilibrium objects, and thus phase diagrams constructed in their presence obviously suffer from severe metastabilities. As recognized by one group of authors in the field, the description of phase behavior in the presence of irreversible protein aggregates should be referred to as "state diagram" rather than phase diagram. ${ }^{17}$

While "state diagrams" have direct relevance to processed fibers dispersion, our main objective in this work was to study systems made only of $\beta$-lactoglobulin fiber aggregates, thus reflecting the true thermodynamic behavior of $\beta$-lactoglobulin amyloid fibers in water. In order to do so, we developed a preparation protocol based on controlled evaporation of water starting from $\beta$-lactoglobulin fiber dispersions of identical initial

(8) Gabriel, J.-C. P.; Davidson, P. Adv. Mater. 2000, 12, 9-20.

(9) Bromley, E. H. C.; Krebs, M. R. H.; Donald, A. M. Faraday Discuss. 2005, 128, 13-27.

(10) Donald, A. M. Soft Matter 2008, 4, 1147-1150.

(11) Aymard, P.; Nicolai, T.; Durand, D.; Clark, A. H. Macromolecules 1999, $32,2542-2552$.

(12) Kavanagh, G. M.; Clark, A. H.; Ross-Murphy, S. B. Int. J. Biol. Macromol. 2000, 28, 41-50.

(13) van der Linden, E.; Venema, P. Curr. Opin. Colloid Interface Sci. 2007, 12, $158-165$.

(14) Krebs, M. R. H.; Devlin, G. L.; Donald, A. M. Biophys. J. 2007, 92, 13361342.

(15) Jung, J.-M.; Savin, G.; Pouzot, M.; Schmitt, C.; Mezzenga, R. Biomacromolecules 2008, 9, 2477-2486.

(16) Veerman, C.; Ruis, H.; Sagis, L. M. C.; van der Linden, E. Biomacromolecules 2002, 3, 869-873.

(17) Bolder, S.; Hendrickx, H.; Sagis, L. M. C.; van der Linden, E. J. Agric. Food Chem. 2006, 54, 4229-4234.

(18) Gosal, W. S.; Clark, A. H.; Ross-Murphy, S. B. Biomacromolecules 2004, 5 , 2408-2149.

(19) Arnaudov, L. N.; de Vries, R.; Ippel, H.; van Mierlo, C. P. M. Biomacromolecules 2003, 4, 1614-1622.

(20) Schokker, E. P.; Singh, H.; Pinder, D. N.; Creamer, L. K. Int. Dairy J. 2000, $10,233-240$

(21) Sagis, L. M. C.; Veerman, C.; van der Linden, E. Langmuir 2004, 20, 924 927.

(22) Renard, D.; Lefebvre, J. Int. J. Biol. Macromol. 1992, 14, 287-291.

(23) Krebs, M. R. H.; Domike, K. R.; Cannon, D.; Donald, A. M. Faraday Discuss. 2008, 139, 265-274. composition. The resulting system is fundamentally different from those previously investigated because it contains a virtually zero content of spherulites and because the fractal topology of the $\beta$-lactoglobulin fibers is identical at all the different concentrations explored.

In order to further assess the effect of the length of the $\beta$-lactoglobulin amyloid fibers, we divided the study into two main classes of fibers: (i) a first class, in which the contour length of the fiber is much larger than their persistence length, leading to objects comparable to semiflexible polyelectrolytes, and (ii) a second class, in which the contour length of the fiber is smaller than their persistence length, leading to true rodlike charged fibers in water. The isotropic-nematic phase transitions of these two systems were determined experimentally, by macroscopic and microscopic observation under polarized light. These results were compared, for long and short fibers, respectively, to the theoretical results predicted using the original Onsager's theory ${ }^{3}$ and same theory modified for semiflexible objects. ${ }^{5}$ The phase diagrams of both systems were then investigated by changing the concentration and the $\mathrm{pH}$. The main picture which emerges from the present study is that $\beta$-lactoglobulin amyloid fibers constitute a system whose complexity encompasses by far that of systems which can be interpreted on the basis of Onsager theories. With the support of cross-polarized optical microscopy and transmission electron micrographs, the sources for such a complexity are extensively discussed, and valuable hypotheses explaining the complex phase behavior of the $\beta$-lactoglobulin are formulated accordingly.

\section{Experimental Section}

2.1. Materials. BioPURE- $\beta$-lactoglobulin (lot JE 003-6-922, from 23-05-2006) was obtained from Davisco Foods International, Inc. (Le Sueur, MN). The powder consisted of $97 \%$ protein (dry basis), among which $95.9 \%$ of $\beta$-lactoglobulin (variant A, $55.6 \%$; variant $\mathrm{B}, 44.4 \%$ ) as determined by HPLC (data not shown). In order to remove non-"native" proteins, the following procedure was set up. First, the protein powder was dissolved in Milli-Q water at $10 \mathrm{wt} \%$ concentration, and the solution was adjusted to $\mathrm{pH} 4.6$, using a $1 \mathrm{M} \mathrm{HCl}$ solution. Then, it was centrifuged at $15000 \mathrm{rpm}$ for $15 \mathrm{~min}$ at $20^{\circ} \mathrm{C}$ using a Sorvall Evolution RC high speed centrifuge (Rotor SLA-1000), and the supernatant was recovered and adjusted to $\mathrm{pH} 2$, using a $1 \mathrm{M} \mathrm{HCl}$ solution. To further remove possible residual traces of insoluble proteins, the supernatant was filtered through a $0.22 \mu \mathrm{m}$ Millipore filter. In order to remove traces of ions that affect $\beta$-lactoglobulin aggregation, ${ }^{24}$ the filtered protein solution was dialyzed first against $\mathrm{pH} 2$ Milli-Q water and second against Milli-Q water, using a Spectra-Por Dialysis Membrane 1, with a MWCO of 6000-8000 Da (Spectrum Laboratories, Inc., Los Angeles, CA). Dialysis tubes were previously boiled for $10 \mathrm{~min}$ in demineralized water in the presence of $1 \mathrm{mM}$ EDTA and extensively rinsed with demineralized water. The volume ratio between the solvent and the protein solution was kept constantly at around 40 during the dialysis. The dialysis was performed at $4{ }^{\circ} \mathrm{C}$ with at least $4 \mathrm{~h}$ between the dialysis buffer changes. After dialysis, the solution was adjusted back to $\mathrm{pH} 2$. The mineral composition of $\beta$-lactoglobulin was determined before and after dialysis by ICP-EAS in a previous work. ${ }^{15}$ For storage the solution was freeze-dried and placed in a desiccator at room temperature. All the experiments were carried out using this dialyzed and freezedried $\beta$-lactoglobulin powder.

2.1.1. Preparation of Long $\beta$-Lactoglobulin Fibers. Dialyzed and freeze-dried $\beta$-lactoglobulin powder was dissolved in Milli-Q water at room temperature, centrifuged at $10800 \mathrm{~g}$ over a

(24) Majhi, P. R.; Ganta, R. R.; Vanam, R. P.; Seyrek, E.; Giger, K.; Dubin, P. L. Langmuir 2006, 22, 9150-9159. 
Table 1. Experimental Conditions for the Production of Heat-Induced Rodlike $\boldsymbol{\beta}$-Lactoglobulin Aggregates

\begin{tabular}{ll}
\hline$\beta$-lactoglobulin concentration $\left(\right.$ wt $\left.\%^{a}\right)$ & 2 \\
$\mathrm{pH}$ & 2.0 \\
temperature $\left({ }^{\circ} \mathrm{C}\right)$ & 90 \\
volume $(\mathrm{mL})$ & 100 \\
stirring & yes \\
heating time $(\mathrm{min})$ & 300 \\
conversion rate $(\%)$ & $75 \pm 3$
\end{tabular}

${ }^{a}$ Since we determined the density of the rods as being 1.002 , we will evenly consider the mass fraction and the volume fraction. For practical reasons, we always worked in mass fraction.

period of $1 \mathrm{~h}$ at $20^{\circ} \mathrm{C}$ using a Sorvall RC3C Plus centrifuge (DuPont, Newtown, CT), adjusted to $\mathrm{pH} 2$, and filtered through a $0.45 \mu \mathrm{m}$ Millipore filter before heat treatment. The experimental conditions we use in this work were adapted from a previously developed protocol ${ }^{15}$ and are summarized in Table 1.

The conversion rate is the amount of $\beta$-lactoglobulin monomers that have been converted into aggregates after heating. ${ }^{16}$ The protocol for its determination was adapted from the protocol of Veerman et al. ${ }^{16}$ and was detailed in a previous work. ${ }^{15}$ We chose to work with $2 \mathrm{wt} \%$ solutions, as at this concentration a high conversion of $\beta$-lactoglobulin monomers into rodlike aggregates can be achieved while avoiding formation spherulites, typically appearing in competition with fiber formation during the heating process at somewhat, larger concentrations. ${ }^{10,23,25}$ As anticipated, these spherulites are irreversible supramolecular aggregates and do not dissemble upon dilution, and their size and number increase with the concentration. ${ }^{17,21}$

The $2 \% \beta$-lactoglobulin solution was distributed in $100 \mathrm{~mL}$ flasks, which were hermetically sealed and placed in a water bath for heating. Each flask contained a magnetic bar to stir the solution during the heating process, as stirring enhanced the conversion rate, the birefringence, and reduced formation of spherulites. ${ }^{26}$ After heating, the flasks were immediately cooled by immersion in ice-water mixtures to quench the aggregation process. The solution was checked by eye for transparency and absence of spherulites. Optical microscopy under polarized light was used to verify a negligible number and size of spherulites.

2.1.2. Preparation of Short $\beta$-Lactoglobulin Rods. A solution of long $\beta$-lactoglobulin fibers, prepared as mentioned above, was diluted to $1 \mathrm{wt} \%$ with $\mathrm{pH} 2$ Milli-Q water and homogenized at $600+100$ bar and five cycles using a two-stage high-pressure homogenizer (Panda 2K, Niro Soavi, Italy). This process enabled to obtain very short fibers from the long ones, with the same cross section.

2.2. Methods. 2.2.1. Optical Microscopy/Cross-Polarizers. The macroscopic aspect of the samples was inspected first by naked eye under normal light to evaluate the transparency/turbidity and determine an eventual macroscopic phase separation. The samples were also visualized between crosspolarizers for birefringence. Optical microscopy under white light and polarized light was then employed to confirm the macroscopic observation and to determine eventual phase boundaries among coexisting phases.

2.2.2. Transmission Electron Microscopy. A drop of the $\beta$-lactoglobulin fibers solution was casted onto a carbon support film mounted on a copper grid. The excess solution was removed after $30 \mathrm{~s}$ using a filter paper. Contrast to electrons was achieved by negative staining by adding a droplet of phosphotungstic acid solution $1 \mathrm{wt} \%$ (PTA, pH 7, Sigma-Aldrich, Switzerland) onto the grid, over a period of $15 \mathrm{~s}$, after deposition of $\beta$-lactoglobulin fibrils solution. Any excess of staining agent was removed again by a filter paper. Electron micrographs were acquired on a CCD

(25) Krebs, M. R. H.; Bromley, E. H. C.; Rogers, S. S.; Donald, A. M. Biophys J. 2005, 88, 2013-2021.

(26) Bolder, S. G.; Sagis, L. M. C.; Venema, P.; van der Linden, E. J. Agric. Food Chem. 2007, 55, 5661-5669.
Table 2. Comparison of Experimental Results with Theoretical Results for the Isotropic-Nematic Phase Transition of Long $\beta$ Lactoglobulin Fibers at Different pH Values

\begin{tabular}{lllll}
\hline & $\begin{array}{c}\text { overall } \\
\text { charge per } \\
\text { monomer }^{27}\end{array}$ & $\begin{array}{c}\text { effective } \\
\text { diameter } \\
(\mathrm{nm})(\text { eq 3) }\end{array}$ & $\begin{array}{c}\text { theoretical } \\
\text { concentration, \% } \\
\left(L_{\mathrm{p}}=1980 \mathrm{~nm}\right)(\mathrm{eq} 2)\end{array}$ & $\begin{array}{c}\text { experimental } \\
\text { result, \% }\end{array}$ \\
\hline 1 & +21 & 17.91 & 5.4 & 0.3 \\
1.5 & +21 & 17.91 & 5.4 & 0.4 \\
2 & +20 & 17.64 & 5.3 & 0.4 \\
2.5 & 19 & 17.37 & 5.2 & 0.4 \\
3 & +16.5 & 16.60 & 5.0 & 0.3 \\
3.5 & +13.1 & 15.34 & 4.6 & 0.3 \\
3.8 & +10 & 13.87 & 4.2 & 0.5
\end{tabular}

camera using a Philips CM100 Biotwin transmission electron microscope operating at $80 \mathrm{kV}$.

2.2.3. Static Light Scattering. Because the long fibers, due to their large sizes, have their Guinier regime in a reciprocal space which is not accessible, static light scattering was performed only the solutions of short $\beta$-lactoglobulin rods. The range of investigated concentrations was between 0.1 and $0.5 \mathrm{wt} \%$. The solutions were filtered through $0.45 \mu \mathrm{m}$ Millipore membrane filters and were gently centrifuged at $3000 \mathrm{rpm}$ for $10 \mathrm{~min}$ before the measurements. These transparent solutions were investigated using an ALV-5000 fast correlator in combination with a monomode fiber compact goniometer system and an argon-ion laser Coherent Innova $308(\lambda=532 \mathrm{~nm})$. The range of scattering vectors covered was $3 \times 10^{-3} \mathrm{~nm}^{-1}<q<10^{-2} \mathrm{~nm}^{-1}$, with $q=$ $4 \pi n / \lambda_{0} \sin (\theta / 2), \lambda_{0}\left(\lambda=\lambda_{0} / n\right), n$, and $\theta$ being respectively the light source wavelength in vacuum, the solvent refractive index, and the angle between the detector and the incident beam. Measurements were made in an angular region from $45^{\circ}$ to $120^{\circ}$ with a step of $5^{\circ}$. Freshly distilled and filtered toluene was used to calibrate the instrument. The Rayleigh ratio for toluene was $3.16 \times 10^{-5} \mathrm{~cm}^{-1}$ at $532 \mathrm{~nm}$. A refractive index increment of $0.189 \mathrm{~mL} \mathrm{~g}^{-1}$ was used. $^{27}$ The data were analyzed using the Zimm procedure as explained in a previous work ${ }^{15}$ in order to extract the weightaveraged molecular weight $M_{\mathrm{w}}$ of the short $\beta$-lactoglobulin fibers.

2.2.4. Determination of the Isotropic-Nematic Phase Transition. The determination of the isotropic-nematic phase transition was carried out by progressively diluting or concentrating the initial solution of $\beta$-lactoglobulin fibers at $\mathrm{pH} 2$, made a priori using the protocol detailed in sections 2.1.1 and 2.1.2, for the long and the short fibers, respectively, depending on whether the initial solution was birefringent or not. The solution was first visualized between cross-polarizers. If the solution was birefringent, it was diluted at constant $\mathrm{pH}$, until disappearance of birefringence. If the initial solution was not birefringent, it was concentrated while maintaining the $\mathrm{pH}$ fixed, until birefringence appeared.

Therefore, starting from the initial solution of long $\beta$-lactoglobulin fibers of $2 \%$ at $\mathrm{pH} 2$, a series of concentrations from 0.1 to $2 \mathrm{wt} \%$ was obtained by dilution with $\mathrm{pH} 2$ Millipore water. The $\mathrm{pH}$ was always checked and adjusted accordingly.

Starting from the initial solution of short $\beta$-lactoglobulin rods of $1 \%$ at $\mathrm{pH} 2$, a series of concentrations from $1 \%$ to $7 \% \mathrm{wt}$ was obtained by a gentle concentration method (see section 2.2.5). Again, the $\mathrm{pH}$ was always checked and adjusted if needed.

The effect of $\mathrm{pH}$ on the isotropic-nematic phase transition was also investigated. The investigated $\mathrm{pH}$ values ranged from 1 to 3.8 , corresponding to a decrease in the fibers' overall charge (per monomer) from +21 to $+10^{27}$ (see Table 2). The protocol followed to determine the isotropic-nematic transition was identical to what described above, apart from the different $\mathrm{pH}$.

2.2.5. Building the Phase Diagram of the $\beta$-Lactoglobulin Fibers in Water. The effects of $\mathrm{pH}$ and concentration were investigated in large ranges in order to construct the phase

(27) Renard, D. Université de Nantes, France, 1994. 
diagrams of long and short $\beta$-lactoglobulin fibers in water. The method followed to build the phase diagram consisted in progressively changing the $\mathrm{pH}$ values starting from a fixed given concentration. We started from $100 \mathrm{~mL}$ of $\beta$-lactoglobulin fibers solution and progressively increased the $\mathrm{pH}$ by $0.5-1$ unit by adding one or two drops of $1 \mathrm{M} \mathrm{NaOH}$, so that the dilution effect was negligible.

Starting from the initial solution of long $\beta$-lactoglobulin fibers of $2 \%$ at $\mathrm{pH} 2$, solutions of $100 \mathrm{~mL}$ of $0.1-2 \mathrm{wt} \%$ (step of 0.5 ) were prepared by dilution with Milli-Q water at $\mathrm{pH} 2$. For one given concentration, the $\mathrm{pH}$ was adjusted to $\mathrm{pH} 2.7 \mathrm{~mL}$ was collected in a glass tube and placed between cross-polarizers. The $\mathrm{pH}$ was then increased by $0.5-1$ unit, and again $7 \mathrm{~mL}$ was collected and placed between cross-polarizers, and the procedure was reiteratively reproduced to cover the entire $\mathrm{pH}$ range from $\mathrm{pH}$ 2 to 7.5 for each concentration. Because there was a critical $\mathrm{pH}$ range, around 5-6, where the fibers precipitated, making the redissolution of the fibers difficult afterward, we also prepared $50 \mathrm{~mL}$ of $\beta$-lactoglobulin fibers solutions that was immediately brought to a basic $\mathrm{pH}$ value, jumping the critical region and avoiding the precipitation, by a quick addition of several drops of $\mathrm{NaOH}(1 \mathrm{M})$. The $\mathrm{pH}$ was then decreased by $0.5-1$ unit by addition of one or two drops of $\mathrm{HCl}(1 \mathrm{M})$. With this procedure we covered the $\mathrm{pH}$ range from 7.5 to 6 . This approach was necessary in order to avoid aggregation and thus construct the correct phase diagram, especially for $\mathrm{pH}$ values above the critical region. Indeed, as we will see later (see section 5), when the fibers were in the critical $\mathrm{pH}$ region around 5-6, they precipitated and their contour length could be measured to decrease to less than $1 \mu \mathrm{m}$, as shown in Figure 10B. The consequence of passing through the critical $\mathrm{pH}$ region would then be the irreversible decrease in fibers length, leading to strong perturbations of the phase diagram. By jumping this critical $\mathrm{pH}$ region allowed us establishing reliable phase diagrams both above and below the isoelectric point of the proteins.

In order to investigate concentrations higher than the concentration of the initial $\beta$-lactoglobulin fibers solution $(2 \%$ for the long fibers and $1 \%$ for the short rods), a gentle and athermal concentration method was developed. This consisted in a nitrogen flux circulating over the solution to concentrate, which dries the water vapor forming at its surface. The solution was poured in a large beaker (at least twice the volume of the solution) which was hermetically covered with a two-hole top, one for the nitrogen flux input and one for air output. The beaker was placed onto a plate stirrer (Vibramax 110, Heidolph Instruments, Germany) and was kept in agitation in order to avoid the formation of gel crowns on the surface during the drying process. We stress here that without agitation irreversible aggregates (highly birefringent under polarized light) form on the beaker surface. This gel being not redispersible in water, it irreversibly affects the final phase diagram. Using the procedure described above, formation of irreversible gel at the beaker surface could be systematically avoided. For each concentration obtained, the $\mathrm{pH}$ effect was screened from $\mathrm{pH} 2$ to 7.5 as described above.

All the samples investigated were kept in sealed $10 \mathrm{~mL}$ glass tubes. Although the phase diagram is expected to be athermal, points were always collected at room temperature. Samples were then stored at $4{ }^{\circ} \mathrm{C}$ to prevent bacterial contamination and observed reiteratively (at room temperature again) for a period of over 2 months to make sure that acquired data corresponded to a thermodynamic equilibrium state. This precaution was especially necessary for samples which showed flow-induced birefringence and needed some relaxation time to become isotropic again as well as for samples which were very viscous.

\section{Theoretical Background}

3.1. Onsager's Theory for Rodlike Particles. On the basis of purely excluded volume considerations, Onsager predicted the isotropic-nematic phase transition for anisotropic particles subjected to hard-core interactions in a solvent to occur at a volume fraction $x:^{3}$

$$
x=4 \frac{D}{L}
$$

with $D$ the diameter of the rod and $L$ the length of the rod.

More precisely, eq 1 gives the so-called "bifurcation point" which is the point at which a nematic phase is first observed coexisting with an isotropic phase $(\mathrm{I}+\mathrm{N})$. By further increasing concentration over a small coexistence concentration window, the biphasic I $+\mathrm{N}$ mixture changes into a purely nematic phase.

Onsager further extended his theory to charged rigid particles, by replacing the diameter $D$ by an effective diameter $D_{\text {eff }}$, taking into account the electrostatic repulsion between the particles and their surrounding double layers (see eq 3 ).

Odijk's et al. ${ }^{4}$ completed Onsager's theory for the charged particles with the addition of the twisting parameter, taking into account the orientation of the rods, and thus the concentrationdependent repulsive interactions of the particles, which may differ from the isotropic and nematic phase.

Accounting for flexibility of particles, was another important achievement ${ }^{5,28}$ which extended Onsager's theory's applicability, since many real anisotropic particles do show some degree of flexibility. The theory was therefore adapted to semiflexible chains (contour length $L \gg$ persistence $L_{\mathrm{p}} \gg$ diameter $D$ ), which could be considered as locally stiff rods only at length scales $<L_{\mathrm{p}}$. In this case, the persistence length $L_{\mathrm{p}}$ rather than the contour length $L$ becomes the characteristic length to be used in the calculation of the phase transition (see eq 2).

3.2. Theoretical Calculation for Systems of $\beta$-Lactoglobulin Fibers in Water. The isotropic-nematic phase transition was calculated using eq $2,{ }^{5}$ for the long $\beta$-lactoglobulin fibers, which are charged and semiflexible:

$$
x=6 \frac{D_{\text {eff }}}{L_{\mathrm{p}}}
$$

with $D_{\text {eff }}$ the effective diameter (see eq 3 ) and $L_{\mathrm{p}}$ the persistence length. The coefficient 6 in front of the term $D_{\text {eff }} / L_{\mathrm{p}}$ is somewhat dependent on the algorithm followed to calculate the bifurcation point and can slightly vary (ca. 10\%) from method to method. More details are given in ref 5 . As will be shown later, however, this is nearly irrelevant to draw the main conclusions of the present article.

The Onsager's effective diameter is

$$
D_{\text {eff }}=D+k^{-1}\left(\ln A+C+\ln 2-\frac{1}{2}\right)
$$

with $D$ the diameter of the rod, $k^{-1}$ the Debye length, $C$ Euler's constant $(C=0.577)$, and $A$ given by Stroobants et al. ${ }^{4}$

$$
A=2 \pi v_{\text {eff }}^{2} k^{-1} Q \exp (-k D)
$$

with $v_{\text {eff }}$ the linear charge density of the rod (see eq 5), $k^{-1}$ the Debye length (see eq 6), and $Q$ the Bjerrum length (see eq 7).

The linear charge density $v_{\text {eff }}$ gives the repartition of the charges along the rod and is expressed by the number of charges per unit length calculated as follows:

$$
v_{\text {eff }}=\frac{\text { charge }_{\text {total }}}{\text { length }_{\text {monomer }}}
$$

(28) Khokhlov, A. R.; Semenov, A. N. Phys. A (Amsterdam, Neth.) 1981, 108, $546-556$. 

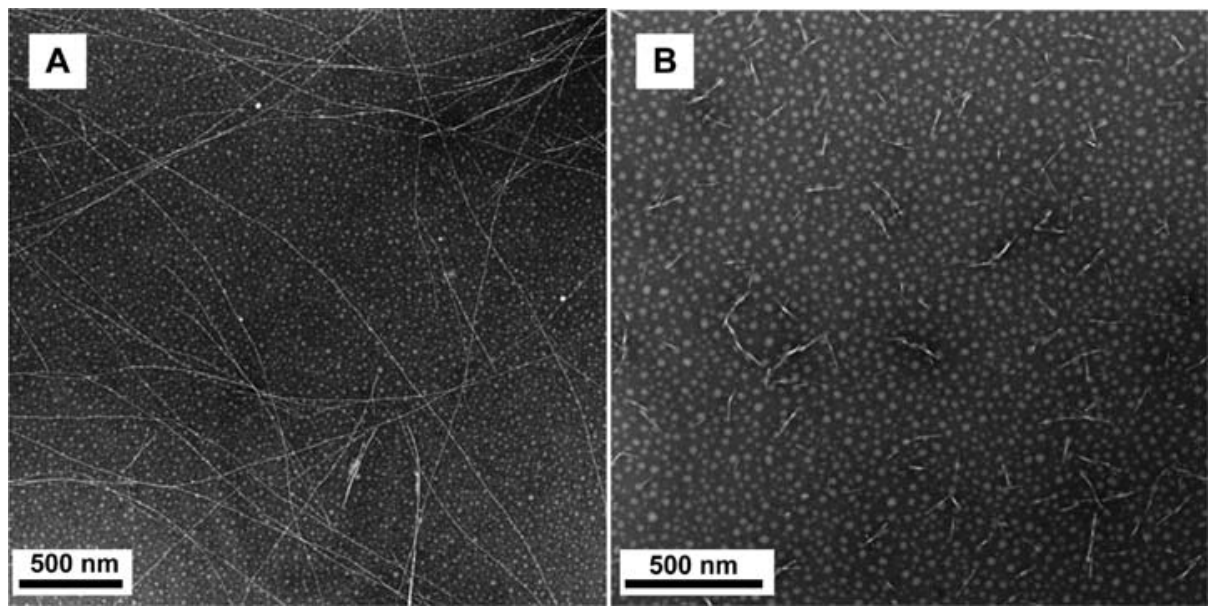

Figure 1. TEM micrographs of heat-induced $\beta$-lactoglobulin fibers diluted to $0.1 \%$ in water at $\mathrm{pH} 2$ : (A) long fibers and (B) short fibers.

The Debye length $k^{-1}$ is the distance over which significant charge screening occurs and is calculated from

$$
k^{2}=\frac{8 \pi N e^{2}}{e_{0} e_{r} K_{\mathrm{B}} T}
$$

with $N$ Avogadro's constant $\left(6.02 \times 10^{23}\right)$, e elementary charge $\left(e=1.6 \times 10^{-19} \mathrm{C}\right), e_{0}$ dielectric permittivity in vacuum $\left(e_{0}=\right.$ $\left.8.85 \times 10^{-12} \mathrm{~F} / \mathrm{m}\right), e_{\mathrm{r}}$ dielectric permittivity in water $\left(e_{\mathrm{r}}=80.1\right)$, $K_{\mathrm{B}}$ Boltzmann's constant $\left(1.38 \times 10^{-23} \mathrm{~J} / \mathrm{K}\right)$, and $T$ absolute temperature $(293 \mathrm{~K})$. Here, considered the lack of salt, $k^{-1}$ was equal to $2.72 \mathrm{~nm}$.

The Bjerrum length $Q$ is the separation length at which the electrostatic interaction between two elementary charges is comparable in magnitude to the thermal energy scale, $K_{\mathrm{B}} T$. It is calculated as follows:

$$
Q=\frac{e^{2}}{4 \pi e_{0} e_{\mathrm{r}} K_{\mathrm{B}} T}
$$

Here, $Q$ is equal to $0.712 \mathrm{~nm}$ (for water at room temperature). Concerning the short rods, which are charged and assumed to be purely rigid, the isotropic-nematic transition was calculated using the formula ${ }^{5}$

$$
x=4 \frac{D_{\text {eff }}}{L_{\mathrm{w}}}
$$

where $D_{\text {eff }}$ is the effective diameter of the rod and $L_{\mathrm{w}}$ is the weightaveraged contour length.

\section{Results}

4.1. Characterization of the Long and Short $\beta$-Lactoglobulin Fibers. The aggregation of $\beta$-lactoglobulin monomers into amyloid fibers is controlled by a very complex series of mechanisms, in which partial unfolding, intermolecular interactions, supramolecular aggregation, and hydrolysis all take place to a different extent, depending on the exact heat denaturation protocol. For example, Bolder et al. ${ }^{30}$ showed that after 5 h of heating at $80{ }^{\circ} \mathrm{C} \beta$-lactoglobulin still existed as monomers, and no products from hydrolysis were present yet. Low-molecularweight hydrolyzed materials would appear after $10 \mathrm{~h}$ of heating at $80{ }^{\circ} \mathrm{C}$, with no more residual native monomers detectable. Moreover, the same group has shown that heating longer than

(29) Rogers, S. S.; Venema, P.; Sagis, L. M. C.; van der Linden, E.; Donald, A M. Macromolecules 2005, 38, 2948-2958.

(30) Bolder, S. G.; Vasbinder, A. J.; Sagis, L. M. C.; van der Linden, E. Int. Dairy J. 2007, 17, 846-853.
$10 \mathrm{~h}$ at $80^{\circ} \mathrm{C}$ could induce hydrolysis of the fibers, as shown by a decrease in conversion experiments, birefringence measurements, and viscosity measurements. ${ }^{26}$ Akkermans et al. ${ }^{31}$ have further shown that $\beta$-lactoglobulin fibers made at $85^{\circ} \mathrm{C}$ during $20 \mathrm{~h}$ were made from hydrolyzed peptides and not intact monomers. Because the amount and nature of intermolecular interactions among the different units of the fibers primarily depend on the constituent secondary structure, it is clear that these results indicate that also the structure of the final fibers is highly dependent on the exact heat denaturation process followed. From the protocol given in section 2.1.1, we were able to obtain very long and thin rodlike $\beta$-lactoglobulin fibers, as shown in the TEM micrograph of Figure 1A. These $\beta$-lactoglobulin fibers showed rigidity between $500 \mathrm{~nm}$ and $\sim 1 \mu \mathrm{m}$ length scale and exhibited certain flexibility at higher length scale. This was in accordance with the results of our previous work, ${ }^{15}$ where by using ultrasmallangle light scattering and static light scattering, we estimated the persistence length of the fibrils to be between 380 and $1980 \mathrm{~nm}$ and showed that these fibers were rigid at length scales below the persistence length but had semiflexible behavior at a larger length scales. The cross section of the fibers observed by TEM were monodisperse and in agreement with our previous statistic measurement, where we determined a cross section of $5.2 \pm$ $1.1 \mathrm{~nm} .{ }^{15}$ The contour length of these fibers was polydisperse, ranging from 1 to $20 \mu \mathrm{m}$. All these values were in good agreement with previous studies on $\beta$-lactoglobulin fibers. $12,16,18,19,21,22,29$

The protocol given in section 2.1.2 enabled us to obtain a new system consisting of short $\beta$-lactoglobulin fibers in water. Working under our set of experimental conditions, we obtained short rods of contour length below the persistence length of the long fibers but still with identical cross section, as seen in Figure 1B. Using TEM micrographs, we determined the number-averaged length of the short rods, which was $L_{\mathrm{n}}=111 \pm 56$ nm ( $n=577$ samples). Using the same set of data, we also extracted the weight-averaged length, using $L_{\mathrm{w}}=\sum L_{i}^{2} / \sum L_{i}$, which was $138 \mathrm{~nm}$. Using static light scattering, we determined the weight-averaged molecular weight $M_{\mathrm{w}}$ of the short fibers via the Zimm plot. Assuming that the fibers were linear strings of $\beta$-lactoglobulin monomers, we estimated the weight-averaged length of the short rods using the following formula:

$$
L_{\mathrm{w}}=\frac{M_{\mathrm{w}}}{M_{\mathrm{w}, \text { monomer }}} D_{\text {monomer }}
$$

(31) Akkermans, C.; Venema, P.; van der Goot, A. J.; Gruppen, H.; Bakx, E. J.; Boom, R. M.; van der Linden, E. Biomacromolecules 2008, 9, 1474-1479. 


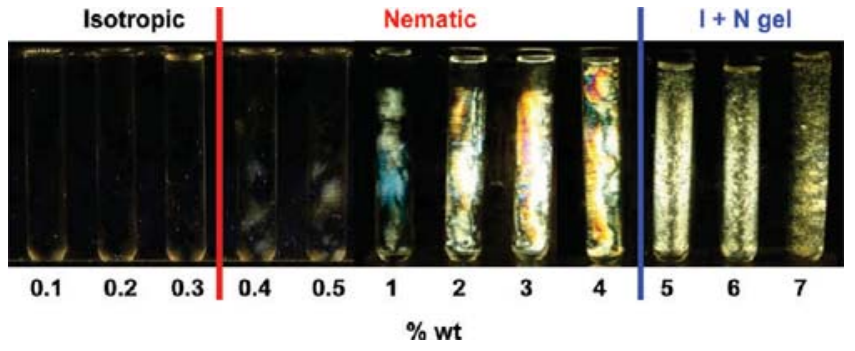

Figure 2. Phase transitions of long $\beta$-lactoglobulin fibers in water at $\mathrm{pH} 2$ and increasing concentrations, as observed under crosspolarized light.

with $M_{\mathrm{w}}$ the weight-averaged molecular weight obtained with the Zimm plot, $M_{\mathrm{w}, \text { monomer }}$ the molecular weight of a single monomer $(18.3 \mathrm{kDa})$, and $D_{\text {monomer }}$ the diameter of a single monomer. With this assumption, $L_{\mathrm{w}}$ was calculated to be $170 \mathrm{~nm}$. This reasonably good agreement with TEM statistic determination can be explained by our heat treatment at $90{ }^{\circ} \mathrm{C}$ and $5 \mathrm{~h}$, for which a very limited hydrolysis of monomers is expected, in agreement with Bolder et al. ${ }^{30}$ Thus, in the present case fibers should be constituted essentially of partially unfolded $\beta$-lactoglobulin monomers with little to nil contribution from hydrolyzed monomers.

4.2. Isotropic-nematic Phase Transition: Experiment vs Theory. We determined in our previous work ${ }^{15}$ that the persistence length of long $\beta$-lactoglobulin fibers should be between 380 and $1980 \mathrm{~nm}$. By using these two values and eq 3, the upper and lower boundary for the isotropic-nematic phase transition can be calculated. By using the lower value of $380 \mathrm{~nm}$ (upper boundary), the concentration at which the isotropic-nematic transition should occur is calculated to occur at $28 \%$, or more, which, as will be shown, is clearly above all the experimental transitions observed in the present systems. The values calculated at different $\mathrm{pH}$ for the isotropic-nematic transition of long $\beta$-lactoglobulin fibers taking $L_{\mathrm{p}}=1980 \mathrm{~nm}$ (lower boundary) are summarized in Table 2.

The experimental isotropic-nematic phase transition was determined visually under polarized light as shown in Figure 2.

Figure 2 presents the series of long $\beta$-lactoglobulin fibers at $\mathrm{pH}$ 2 and increasing concentrations. The solutions were all liquid and transparent up to $5 \%$, where a gel was obtained. Under polarized light, the sample at $0.3 \%$ was not birefringent, whereas at $0.4 \%$ the sample started to be very slightly birefringent. Therefore, the isotropic-nematic phase transition was assigned to occur at $0.4 \%$. The sample became progressively more birefringent as the concentration was increased. At $2 \%$ and above, the sample was birefringent with bright colors, suggesting the possible presence of cholesteric liquid crystalline phases. These could originate, for example, by the formation of chiral helical conformations of the fibers. Circular dichroism was attempted to elucidate this point, but at these concentrations, the intensity of absorbed light was so high to saturate the circular dichroism signal, making impossible drawing of any conclusion.

Optical microscopy was used as complementary technique to characterize the liquid crystalline phase behavior of $\beta$-lactoglobulin fibers in water.

As seen in Figure 3, the isotropic phase was characterized by one uniform continuous phase, which is the same under normal and polarized light. The nematic phase was characterized by one single continuous phase showing birefringent waves spanning the entire sample, as observed under polarized light but not visible under normal light. The gel revealed a microscopic phase separa-

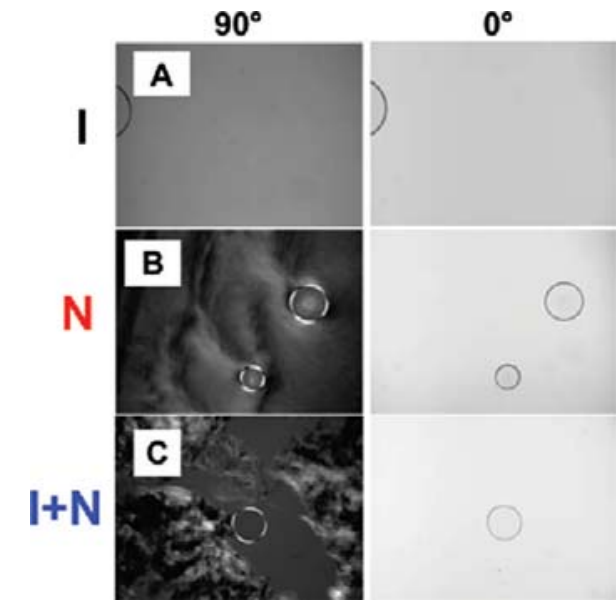

Figure 3. Optical micrographs of long $\beta$-lactoglobulin fibers in water at $\mathrm{pH} 2$ under polarized $\left(90^{\circ}\right)$ and normal light $\left(0^{\circ}\right)$ at different concentrations: (A) $0.2 \%$, (B) $3 \%$, and (C) $5 \%$. These micrographs show respectively an isotropic phase, a nematic phase, and a microseparated biphasic region formed by isotropic plus nematic phases. In $(\mathrm{C})$ the interface separating the isotropic from the nematic phase is only visible under cross-polarized light, disappearing under normal light. This indicates the presence of an interface with only small concentration gradients and only observable under polarized light. The bubble drop is given as a reference of a real interface within two phases with strong density changes (and thus refractive index changes): observable in both polarized and normal light.

tion, with coexisting isotropic (nonbirefringent) and nematic (birefringent) phases, visible under polarized light. Figure $3 \mathrm{C}$ clearly shows that the sharp interfaces between the nematic and the isotropic phases in the gel disappear when cross-polarization is removed. For comparison, the micrographs were acquired in presence of air bubbles to provide a physical interface, which remains visible, under both normal and polarized light.

The isotropic-nematic phase transitions which were experimentally determined depending on the $\mathrm{pH}$ are summarized and compared to the theoretical results in Table 2.

The experimental isotropic-nematic transitions determined at different $\mathrm{pH}$ in the present work (Table 2) are in good agreement with the work of Sagis et al., ${ }^{21}$ who found an isotropic-nematic phase transition of $0.5 \%$ at $\mathrm{pH}$, although in that case, the phase was reported as isotropic plus nematic droplets (spherulites), whereas in the present work, the transition is clearly an isotropic-nematic transition.

Furthermore, our experimental results are in strong disagreement with the results predicted by the theory, for which phase transitions are expected at concentrations which are 1 order of magnitude higher.

Finally, it should be considered that only $75 \%$ of the $\beta$-lactoglobulin contributes to the isotropic-nematic transition, as $25 \%$ of the $\beta$-lactoglobulin native protein is not converted into fibers, as explained before. This contributes to further set apart the theoretical and experimental phase transitions.

The experimental determination of the isotropic-nematic phase transition of short $\beta$-lactoglobulin rods was not obvious in comparison with the long $\beta$-lactoglobulin fibers. Indeed, as shown in Figure 4, under normal light, the samples showed an increase in turbidity with the concentration, and when the same samples were placed between cross-polarizers, it was difficult to clearly disentangle the contribution to birefringence due to a nematic phase versus light diffraction. 


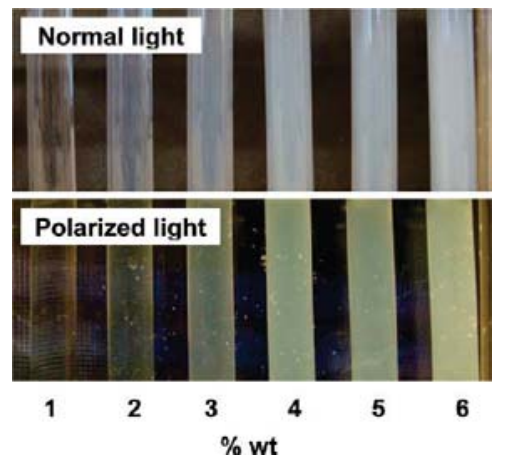

Figure 4. Short $\beta$-lactoglobulin rods at $\mathrm{pH} 2$ and increasing concentrations visualized under normal light and between crosspolarizers.

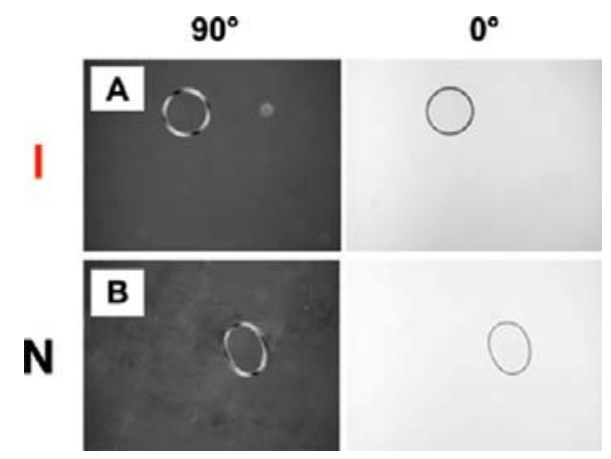

Figure 5. Optical micrographs of short $\beta$-lactoglobulin rods in water at $\mathrm{pH} 2$ under polarized $\left(90^{\circ}\right)$ and normal light $\left(0^{\circ}\right)$ at (A) $2 \%$ and (B) $3 \%$, showing respectively an isotropic phase and a nematic phase.

Table 3. Comparison of Experimental and Theoretical Results for the Isotropic-Nematic Phase Transition of Short $\beta$-Lactoglobulin Rods at pH 2

\begin{tabular}{|c|c|c|c|c|}
\hline $\mathrm{pH}$ & $\begin{array}{c}\text { overall } \\
\text { charge per } \\
\text { monomer }\end{array}$ & $\begin{array}{l}D_{\text {eff }} \\
(\mathrm{nm}) \\
\text { (eq 3) }\end{array}$ & $\begin{array}{c}\text { theoretical } \\
\text { isotropic-nematic } \\
\text { transition, \% } \\
\left(L_{\mathrm{w}}=170 \mathrm{~nm}\right)(\text { eq } 8)\end{array}$ & $\begin{array}{l}\text { experimental } \\
\text { result, } \%\end{array}$ \\
\hline & +20 & 17.64 & 41.5 & 3 \\
\hline
\end{tabular}

Optical microscopy was necessary in order to fully assess the isotropic-nematic phase transition, which, unlike for the long $\beta$-lactoglobulin fibers, was very tenuous for short rods as shown in Figure 5.

Using this procedure, we determined the isotropic-nematic phase transition for short $\beta$-lactoglobulin rods to occur at $3 \%$. Once again, the experimental phase transition was found to occur at concentrations 1 order of magnitude lower than those expected based on Onsager's theory (eq 8, see Table 3).

The fact that the difference between the experiment and the theory was about 1 order of magnitude for both systems suggests that the theory is not as straightforwardly applicable to the present fibers. Possible reasons for these discrepancies will be discussed in the last part of the paper.

4.3. Phase Diagram of $\boldsymbol{\beta}$-Lactoglobulin Fibers in Water. The phase diagram of long $\beta$-lactoglobulin fibers is given in Figure 6 . The phase diagram superimposed the set of results from observation under both normal light and polarized light. The long $\beta$-lactoglobulin fibers exhibited rich and complex phase behavior. The liquid state was found between 0 and $4.5 \mathrm{wt} \%$, and from $5 \%$ and above, a gel was obtained, as previously mentioned (see section 4.2 and Figure 2).
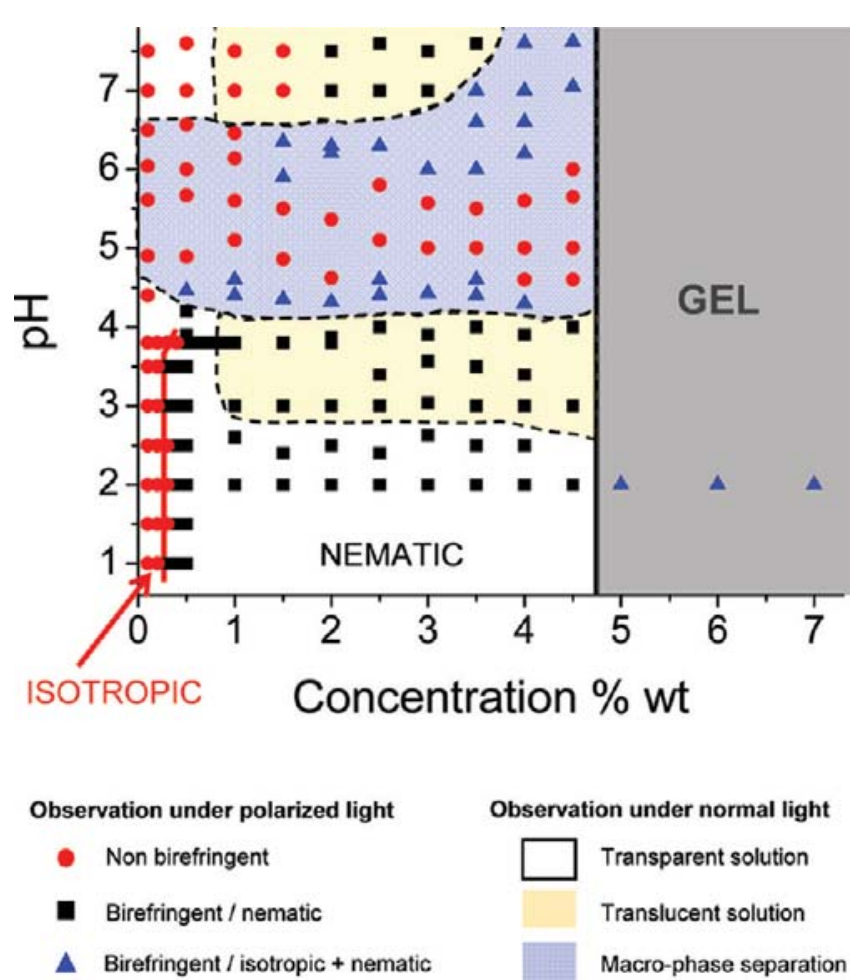

Figure 6. Phase diagram of long $\beta$-lactoglobulin fibers in water.

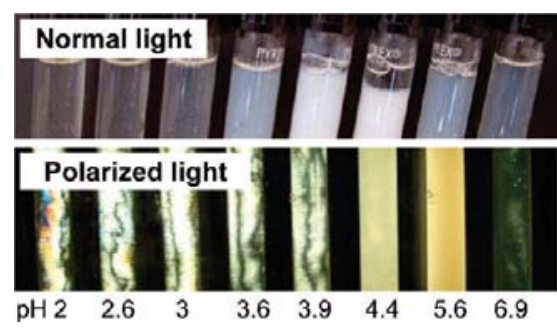

Figure 7. Long $\beta$-lactoglobulin fibers at $3 \%$ and increasing $\mathrm{pH}$ values visualized under normal light and between cross-polarizers.

At macroscopic scale and under normal light, the phase diagram revealed domains of completely transparent solutions, translucent solutions, or phase-separated solutions. For instance, below $\mathrm{pH} 2.7$, the solution of long $\beta$-lactoglobulin fibers remained transparent, at any concentration explored below the gel threshold (white region). A translucent solution was observed for $\mathrm{pH}$ regions between 2.7 and 4.2 and above 7 (yellow region), for concentrations above $1 \%$. A macroscopic phase separation occurred for all concentrations between $\mathrm{pH} 4.2$ and 6.6 (blue region).

Using polarized light and polarized light microscopy, we delimited isotropic, nematic, and biphasic domains. Figure 7 shows a typical series of samples screening the various $\mathrm{pH}$ at the isocomposition concentration of $3 \%$, as observed under normal light and under polarized light.

Referring to the phase diagrams in Figure 6, the nonbirefringent region was observed for two distinct areas (red spots). The first area was observed at any $\mathrm{pH}$ below 4.5 and above 6.5 (that is, far from the protein isoelectric point) and concentrations lower than the corresponding isotropic-nematic transitions. Considering that this region of the phase diagram was completely transparent and nonbirefringent, this indicated the presence of an isotropic phase with individually dispersed protein fibrils. The second one was observed for $\mathrm{pH}$ values between 4.6 and 5.6 for all concentrations below the gel threshold. Because the lack of 

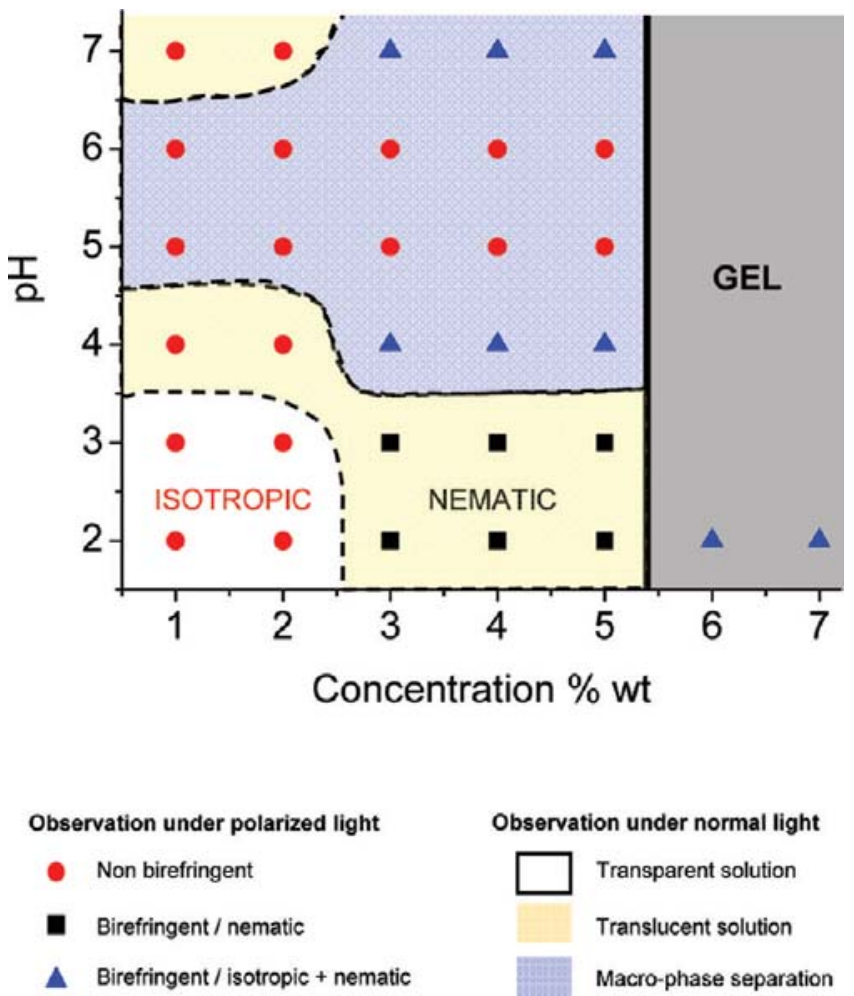

Figure 8. Phase diagram of short $\beta$-lactoglobulin fibers in water.

birefringent behavior was simultaneously observed with turbidity, this region was due to aggregation and precipitation of the fibers, which became globally neutral around the isoelectric $\mathrm{pH}$ of 5.1. Outside the $\mathrm{pH}$ region between 4.2 and 6.6 and above a critical concentration $(0.3 \%$ for $\mathrm{pH}$ below 4.2 and $1.5 \%$ for $\mathrm{pH}$ above 6.6), a very large birefingent domain was observed (black squares and blue triangles), corresponding to the nematic phase of the $\beta$-lactoglobulin fibers.

One interesting observation concerns the macrophase-separated phase diagram region in proximity of the isoelectric point (blue region). In this large window of the phase diagram, two types of phase behavior were observed. Between $\mathrm{pH} 4.6$ and 5.6, i.e., closest to the isoelectric $\mathrm{pH}$, the sample macrophase-separated into transparent supernatant and white opaque sediment, both lacking birefringence under polarized light (red spots). We assumed this behavior to arise from the complete neutralization of charges on the fibers, leading to pronounced random-coil behavior of the fibers followed by isotropic aggregation. At $\mathrm{pH}$ values a bit further from the isoelectric $\mathrm{pH}$, the sample also macrophase-separated into transparent supernatant and white opaque sediment, but the sediment was birefringent under polarized light (blue triangles). We assumed this behavior was due to the partial neutralization of charges in the fibers, which, although aggregating, would preserve a semiflexible behavior with then a more pronounced tendency to align, as also witnessed by the proximity in the phase diagram of the pure nematic phases.

The entire region of the phase diagrams below the gel threshold presented a reversible physical behavior upon $\mathrm{pH}$ and concentration changes, except in the isoelectric region (blue area), confirming the thermodynamic character of the phase diagram. For the gel, however, dissolution of the nematic phase did not occur upon dilution, and the system maintained birefringent spots both between cross-polarizers and under polarized light microscopy. This indicates that the presence of aggregation in the gel region does not follow thermodynamic behavior, but rather a concentration-induced irreversible aggregation of the fibers, similarly to what is observed for the formation of spherulites. A possible explanation on why the birefringent aggregates coexist with an isotropic phase rather than a nematic phase can simply be found on the nonequilibrium nature of this region of the diagram: the gel being obtained by further concentrating the pure nematic region, irreversible aggregation within the nematic phase starts to occur, which phase separates the system into a fiber-enriched region (the aggregates) and a fiber-impoverished region (the isotropic continuous phase obtained by a nematic $\rightarrow$ isotropic shift upon diminution of effective fiber content).

The phase diagram of the short $\beta$-lactoglobulin rods is given in Figure 8 . As expected, the phase diagram was similar to the one for the long $\beta$-lactoglobulin fibers. As explained in section 4.2, due to the difficulty to draw the phase diagram as accurately as for the long $\beta$-lactoglobulin fibers, we investigated the concentration range and the $\mathrm{pH}$ range by an increment step of 1.0.

As for the long $\beta$-lactoglobulin fibers, the phase diagram of the short $\beta$-lactoglobulin rods showed transparent domains, translucent domains, and macrophase-separated domains.

The main difference with the long $\beta$-lactoglobulin fibers concerned the isotropic-nematic phase transition, which occurred at $3 \%$ (see section 4.2). Another difference worth being mentioned is that for concentrations from 3 to $5 \%$ and $\mathrm{pH}$ below 3 , where a nematic phase was observed, the solution was translucent, whereas in the case of long $\beta$-lactoglobulin fibers, the solution remained transparent at these same conditions. We assumed that the short rods had a greater propensity for aggregation, perhaps due to the high-pressure homogenization step or simply the shorter length.

We finally stress again that the real thermodynamic nature of the phase diagrams presented above was assessed by checking in both cases reversibility upon concentration/dilution and changes in $\mathrm{pH}$. Both long and short fiber systems were found to be completely reversible in the liquid domain $(<5 \%$ and $<6 \%$ for the long and short fibers, respectively) and outside the isoelectric region (approximatively below 4.3 and above 6.5 ).

\section{Discussion}

The results presented above highlight the complex phase behavior of $\beta$-lactoglobulin fibers in water and, with respect to the isotropic-nematic transition, a marked deviation between the experimental findings and the behavior expected based on Onsager's theory. We shall discuss here the possible relevant sources for such a complex behavior, from a physical chemistry point of view.

5.1. Formation of $\boldsymbol{\beta}$-Lactoglobulin Fibers Supramolecular Helical Bundles. The first element of complexity may very well arise from the process of fibril formation and conformation. The fibers are made from partially unfolded $\beta$-lactoglobulin monomers, which by heat denaturation are able to form long linear aggregates, held together by intermolecular $\beta$-sheets. ${ }^{9}$ Previous literature, however, has suggested that individual fibers can also associate together into supramolecular helical bundles. ${ }^{32}$ In order to verify this hypothesis in the present system, we took TEM micrographs at various concentrations and $\mathrm{pH}$ for the long and the short $\beta$-lactoglobulin fibers.

Figure 9 shows indeed the presence of such helixes for both long and short $\beta$-lactoglobulin fiber systems, having a pitch usually ranging from 60 to $100 \mathrm{~nm}$. For the long $\beta$-lactoglobulin fibers, the TEM micrographs indicate that increasing the concentration does not significantly increase the number of helixes.

(32) Arnaudov, L. N. Wageningen Universiteit, The Netherlands, 2005. 

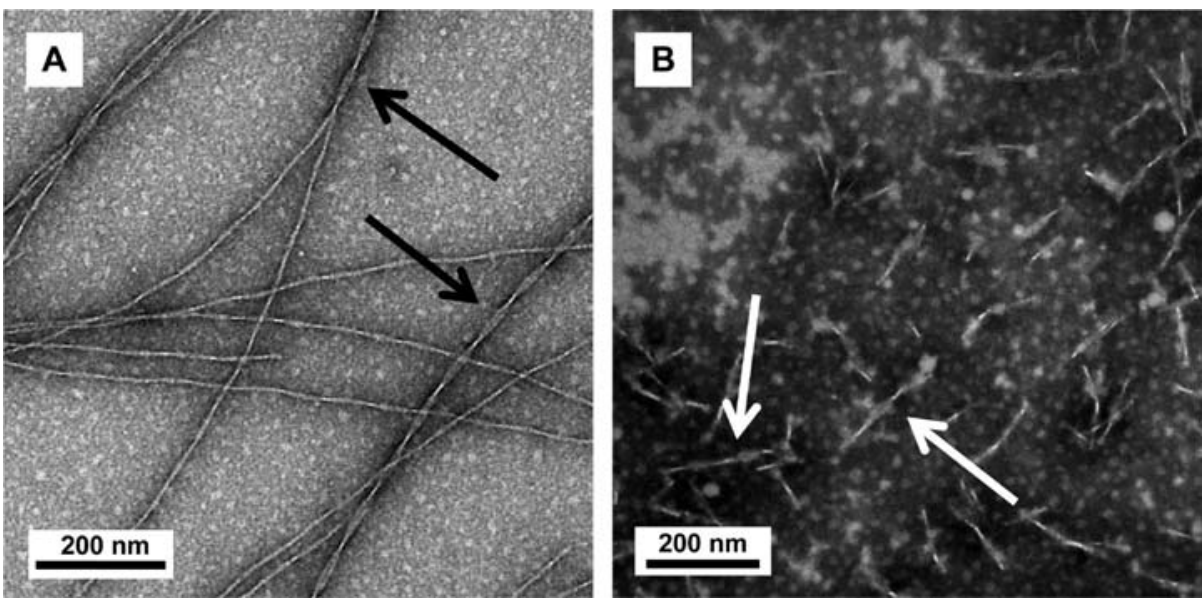

Figure 9. TEM micrographs of helixes in solutions of $\beta$-lactoglobulin (A) long and (B) short fibers. The initial conditions before dilution for TEM observation were (A) $1 \%, \mathrm{pH} 2$ and (B) $5 \%, \mathrm{pH} 3$.
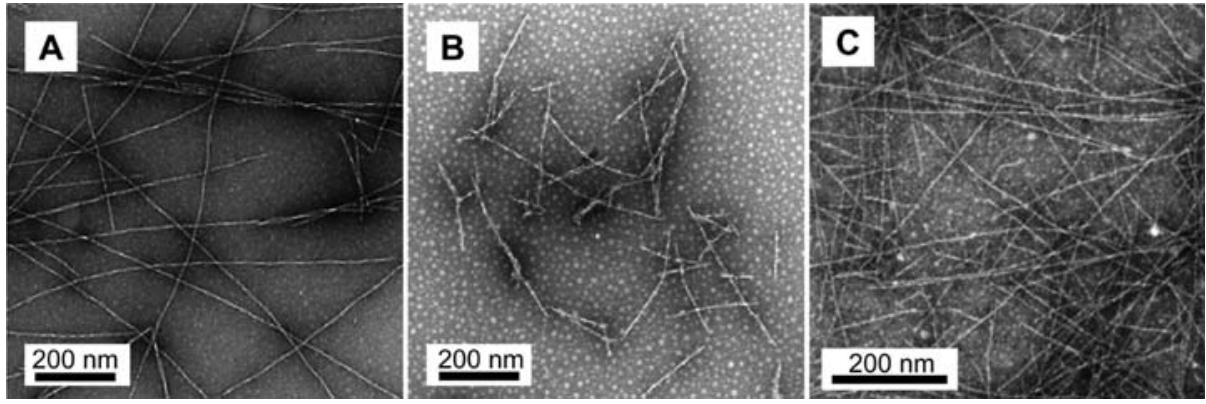

Figure 10. TEM micrographs of long $\beta$-lactoglobulin fibers at different initial concentrations and $\mathrm{pH}$ : (A) $2 \%, \mathrm{pH} 3.8,(\mathrm{~B}) 0.1 \%$, pH 5.6 , and (C) $1.5 \%, \mathrm{pH} 7.5$. For TEM observation the samples were diluted to $0.1 \%$.

However, increasing the $\mathrm{pH}$ from 2 to 4 significantly increased the number of helixes, which might explain why the solution became translucent above $\mathrm{pH}$ 3. Concerning the short $\beta$-lactoglobulin fibers, the presence of helices is greatly enhanced by the concentration even at $\mathrm{pH} 2$, which again may explain the extended translucent region of the phase diagrams with short rods (see Figures 4 and 8). High-pressure homogenization may have favored aggregation, by possibly exposing free binding sites when cutting the fibers into short rods. This may explain the more pronounced tendency of short rods compared to long fibers to produce helices upon increase of concentration. Indeed, by increasing the concentration to $5 \mathrm{wt} \%$ at $\mathrm{pH} 2$, all the short $\beta$-lactoglobulin rods were found in pair forming helixes with one or two pitches of $100 \mathrm{~nm}$ (see Figure 9B).

Obviously, the presence of supramolecular helical aggregates immediately add complexity to the nature of the $\beta$-lactoglobulin fiber systems, as it affects directly both the effective diameter $D_{\text {eff }}$, and the persistence length $L_{\mathrm{p}}$ (and ultimately the contour length $L_{\mathrm{w}}$ ). Since both $D_{\text {eff }}$ and $L_{\mathrm{p}}$ are expected to be increased by the helical formation, it becomes challenging to predict how the isotropic-nematic transition may be affected by this phenomenon.

\subsection{Persistence Length Dependence on Linear Charge} Density. A second major complication arises from the fact that $\beta$-lactoglobulin fibers behave quite differently from standard semiflexible polyelectrolytes. In the case of standard polyelectrolytes, the increase of $\mathrm{pH}$ (and thus the decrease of linear charge density) is expected do simultaneously decrease both $D_{\text {eff }}$ and $L_{\mathrm{p}}$. The conformation of the $\beta$-lactoglobulin fibers, however, does not seem to be very much affected by a change of $\mathrm{pH}$. A screening on the persistence length of the fibers depending on the $\mathrm{pH}$ was carried out by TEM, as shown in Figure 10.

In Figure 10, it can be observed that there is no real striking effect of $\mathrm{pH}$ on the persistence length of the long $\beta$-lactoglobulin fibers, which remains quite extended in all the examples shown. At pH 3.8 (picture A), where the linear charge density is already decreased by half from +20 to +10 , the fibers still maintain a very strong rigidity. At pH 5.6 (picture $\mathrm{B}$ ), where the total linear charge density is nearly zero and the system is in an isotropic, turbid aggregated state, the fibers still maintained a rodlike shape. However, most of them were found to be aggregated together and with a much shorter contour length. At $\mathrm{pH} 7.5$ (picture $\mathrm{C}$ ), the fibers were found again rigid, yet with their original long contour length and a persistence length higher than $500 \mathrm{~nm}$. The fact that the fiber's persistence length was not dramatically affected by the $\mathrm{pH}$, even close to the isoelectric point, was not so surprising, as ionic strength has been shown in the literature to moderately affect the persistence length of these fibers. For example, Aymard et al. ${ }^{11}$ showed using light scattering that $\beta$-lactoglobulin fibers made at $\mathrm{pH} 2$ and $0.013 \mathrm{M}$, heated at $80^{\circ} \mathrm{C}$, had a persistence length of at least $600 \mathrm{~nm}$. When these fibers were dissolved in water at $0.1 \mathrm{M}$, it was shown that the persistence length was decreased to $120 \mathrm{~nm}$, as expected based on the decrease of electrostatic repulsions, but that this persistence length was still much higher than the persistence length of $\beta$-lactoglobulin fibers initially made at $0.1 \mathrm{M}$, that is, $38 \mathrm{~nm}$. This suggests a strong dependence of the persistence length on the initial experimental conditions: if semiflexible fibers are formed at very low ionic strength, their persistence length does not decrease dramatically with a high ionic strength and is not affected by dilution or 


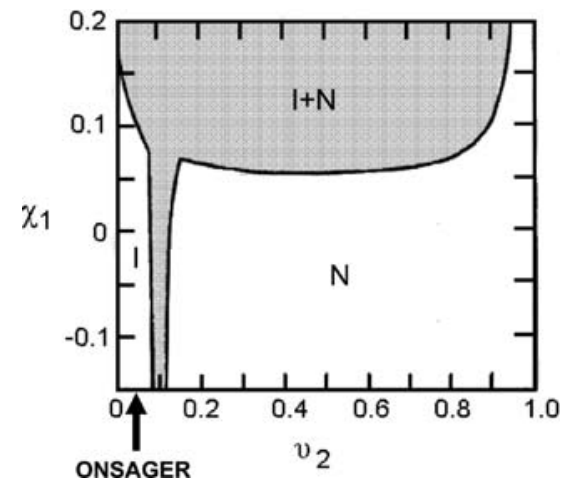

Figure 11. Phase diagram of rod-solvent suspensions considering particle-solvent interactions, as predicted by Flory's lattice theory. The isotropic-nematic phase transition according to Onsager's theory is also mentioned. Redrawn from ref 35.

concentration. ${ }^{11,21}$ It was suggested that other factors, nonpresent in polyelectrolytes, do contribute to the high persistence length of protein amyloid fibers, such as the formation of intermolecular hydrogen-bonding $\beta$-sheets, which would partially maintain the rigidity by interlocking mechanisms. ${ }^{12,29,33}$ Furthermore, changes in $\mathrm{pH}$ have little effect not only on the persistence length but also on the $D_{\text {eff: }}$ this latter is increased on one hand by the $\mathrm{pH}$-promoted helical aggregation; but on the other hand, it is simultaneously decreased by the decrease in linear charge density occurring with the increase of $\mathrm{pH}$ (see eq 3 ). This combination of events may very well explain why the isotropic-nematic transition seems to occur at the same concentrations independently of the $\mathrm{pH}$ (see Figure 6 and Table 2).

5.3. Amphoteric Nature of $\boldsymbol{\beta}$-Lactoglobulin Fibers. The third important factor to bear in mind is that charges are not uniformly distributed along the fibers, that is, the fibers are amphoteric $^{27}$ as the charge distribution depends on the residues of the amino acids forming the $\beta$-lactoglobulin monomer. At $\mathrm{pH}$ $2, \beta$-lactoglobulin fibrils are positively charged $(+20)$ and bear only positive charges; therefore, the electrostatic interactions are purely repulsive. ${ }^{11}$ However, when increasing the $\mathrm{pH}$, the overall positive charge of fibrils starts to decrease while negatively charged residues start to appear. This leads to electrostatic interactions among fibers of the same overall charge, which might not only be purely repulsive: both attractive and repulsive electrostatic interactions, together with hydrophobic interactions, can then start to take place among different patches of the fibers. This amphoteric character of the fibers may very well explain the ability of fibers to form helixes at $\mathrm{pH} 2$, where the repulsion of neutral charges occurs together with (attractive) hydrophobic interactions among neutral residues or at $\mathrm{pH}$ larger than 2 , where both negative and positive charges are present. The fact that the helixes have a regular periodicity is also in strong support of this hypothesis. Work is currently in progress to elucidate the mechanisms of formation of these helices in $\beta$-lactoglobulin fibers.

5.4. Activation of the Free Thiol Group at pH 7. As an additional enlightening example illustrating the difference between $\beta$-lactoglobulin fibers and classical polyelectrolytes, it is interesting to compare the isotropic-nematic transitions measured for long fibers at acidic and basic $\mathrm{pH}$. At $\mathrm{pH} 7$ and above, the fibers bore an overall charge of $-8.5^{27}$ and the isotropic-nematic phase transition occurred at a concentration between 1.5

(33) Gosal, W. S.; Clark, A. H.; Pudney, P. D. A.; Ross-Murphy, S. B. Langmuir 2002, 18, 7174-7181. and $2 \%$ (see Figure 6 ). At $\mathrm{pH} 3.9$, the fibers bore an overall charge of $+8.6^{27}$ and the isotropic-nematic phase transition occurred at $0.5 \%$, which is $3-4$ times lower. If the fibers were classical polyelectrolytes, then according to Onsager's theory, the isotropic-nematic phase transition should occur at exactly the same concentration, the only difference among the two cases being only the sign of the overall charge. The higher transition observed for pH 7 and above may be explained by the activation of the free thiol groups ${ }^{30,34}$ and the possibility to form covalent disulfide bridges between neighbor fibers, leading to random branching among fibers. This would have the effect to decrease the average aspect ratio and thus to delay the isotropic-nematic transition, as observed experimentally.

5.5. Unfavorable Rod-Solvent Interactions and Flory's Theory. One of the unexpected results of the present work was the evidence of hydrophobic interactions between protein fibers and water leading to fibers aggregation and formation of supramolecular helices. In 1956, Flory has published a lattice theory for suspensions of rods in a solvent, which enables to account for positive, unfavorable rod-solvent interactions. ${ }^{35}$ Figure 11 shows a schematic phase diagram illustrating the main findings of Flory's theory and the differences with respect to Onsager's predictions. In athermal conditions, that is, for negative or zero rod-solvent Flory-Huggins interaction parameters $\chi$, Flory's and Onsager's theories basically predict the same trend, which is an isotropic - nematic transition occurring at moderately concentrated compositions and a narrow $\mathrm{I}+\mathrm{N}$ coexistence channel. The difference in the exact compositions of the predicted $\mathrm{I} \rightarrow \mathrm{N}$ transitions for the two theories, by a factor 2, results from the lattice treatment in Flory's case. More interestingly, as soon as positive, unfavorable interactions are occurring among the rods and the solvent, meaning a positive $\chi$ value, the $\mathrm{I}+\mathrm{N}$ channel widens to a very large coexistence region and the isotropic line moves to vanishing small compositions. ${ }^{35}$ This may very well explain why amyloid fibers experiencing hydrophobic interactions with water (and thus interfibers aggregation) would experience an isotropic $\rightarrow$ nematic transition at compositions well below those predicted by Onsager's theory.

Our isotropic-nematic phase study on both semiflexible long fibers and short rodlike fibers has shown for very low $\mathrm{pHs}$ the direct transition from a purely isotropic to a purely nematic phase. Both Onsager's and Flory's theories allow, in correspondence of bifurcation points, for an intermediate region in between the isotropic and the nematic, where the two phases coexist. As just discussed, this coexistence region is small in the case of Onsager theory and is large in Flory's treatment as soon as positive interaction energies are added to the model. Here, despite careful observation under cross-polarized optical microscopy, we have no evidence of interfaces between a nematic and an isotropic phase, if the $\mathrm{pH}$ is maintained low enough ( $\mathrm{pH} 4$ or less for long fibers, $\mathrm{pH} 3$ or less for short fibers). Therefore, if a coexistence region exists in this region of the phase diagram, it necessarily needs to be vanishingly narrow. This is quite in contrast with the isotropic plus nematic region found for both long and short fibers at $\mathrm{pH}$ slightly closer to the isoelectric points (blue triangles in Figures 6 and 8, respectively), for which the region of coexistence seem to extend to a large portion of the phase diagram.

To conclude this study, we have shown that systems made of $\beta$-lactoglobulin fibers in water constitute remarkably complex systems with very rich phase behavior. The complexity of the

(34) Hoffmann, M. A. M.; van Mil, P. J. J. M. J. Agric. Food Chem. 1997, 45, 2942-2948.

(35) Flory, P. J. Proc. R. Soc. London A 1956, 234, 73-89. 
fibers has multiple origins, but we can mention at least two main factors: (i) the intrinsic rigidity of the fibers, which is only partially due to the electrostatic charge density, but also depends on the mechanisms of molecular reorganization of the proteins during heat denaturation, and (ii) the amphoteric nature of the fibers, for which an odd distribution of negative and positive charges along their contour length leads to complex inter- and intrafiber interactions, the most evident being the formation of helices. As a main consequence of this level of complexity, the direct application of conventional Onsager and Onsager-modified theories appear to be inappropriate to fully describe the present systems, and the Flory's theory accounting for hydrophobic interactions of rod suspensions in water seems to produce theoretical predictions which are more consistent with our experimental observations.

Acknowledgment. Nestlé Management is gratefully acknowledged for allowing publication of the present work. R.M. thanks Seth Fraden at Brandeis University for interesting and stimulating discussions. 